Math. Nachr. xxx (2003), 1-14

\title{
Phase dynamics in the real Ginzburg-Landau equation
}

By IAn Melbourne of the University of Surrey and Guido SchneIder of Karlsruhe

(Received June 2002; revised version January 2003)

Abstract. Spatially periodic equilibria $A(X, T)=\sqrt{1-q^{2}} e^{i q X+i \phi_{0}}$ are the locally preferred planform for the Ginzburg-Landau equation $\partial_{T} A=\partial_{X}^{2} A+A-A|A|^{2}$. To describe the global spatial behavior, an evolution equation for the local wave number $q$ can be derived formally. The local wave number $q$ satisfies approximately a so called phase diffusion equation $\partial_{\tau} q=\partial_{\xi}^{2} h(q)$. It is the purpose of this paper to explain the extent to which the phase diffusion equation is valid by proving estimates for this formal approximation.

\section{Introduction}

The (normalized) real Ginzburg-Landau equation

$$
\partial_{T} A=\partial_{X}^{2} A+A-A|A|^{2}
$$

with $X \in \mathbb{R}, T \geq 0$, and $A(X, T) \in \mathbb{C}$, is a universal amplitude equation which is derived by multiple scaling analysis in order to describe bifurcating solutions in pattern forming systems close to the threshold of the first instability. The amplitude $A$ describes slow modulations in space and time of the underlying bifurcating spatially periodic pattern. Examples of such pattern forming systems are reaction-diffusion systems, systems in nonlinear optics, or hydrodynamic stability problems, for example Rayleigh-Bénard convection or the Taylor-Couette problem. A mathematical theory of this reduction to the real (and complex) Ginzburg-Landau equation has been developed by several authors (cf. [CE90, vH91, Sch94, Me98, Me99]). It is nowadays a well established mathematical tool which can be used to obtain new mathematical results (cf. [Sch99]), such as global existence results and uppersemicontinuity of attractors.

The real Ginzburg-Landau equation possesses a family of stationary solutions

$$
A(X, T)=\sqrt{1-q^{2}} e^{i q X+i \phi_{0}}=A_{\mathrm{per}}\left[q, \phi_{0}\right](X)
$$

with $q \in(-1,1), \phi_{0} \in \mathbb{R}$. For $q^{2} \leq 1 / 3$ the equilibria are spectrally stable, whereas for $q^{2}>1 / 3$ they are Eckhaus or sideband unstable [Eck65]. Locally in space, these

1991 Mathematics Subject Classification. Primary: 35A 35; Secondary: 35K55, 35 Q 30.

Keywords and phrases. approximation, phase diffusion, pattern formation. 
solutions are the preferred planform. In order to describe the global behavior in space, an evolution equation for the local wave number $q$ can be derived. Allowing $q$ to vary slowly in time and space, we write

$$
A_{\mathrm{per}}\left[\psi\left(\delta X, \delta^{2} T\right), \phi_{0}\right](X):=\sqrt{1-\left(\psi\left(\delta X, \delta^{2} T\right)\right)^{2}} \exp \left(i \int_{0}^{X} \psi\left(\delta X^{\prime}, \delta^{2} T\right) d X^{\prime}+i \phi_{0}\right)
$$

with $0<\delta \ll 1$ a small perturbation parameter, where $\psi$ satisfies the phase diffusion equation

$$
\partial_{\tau} \psi=\partial_{\xi}^{2} h(\psi)
$$

with $\tau=\delta^{2} T, \xi=\delta X$, and $h: \mathbb{R} \rightarrow \mathbb{R}$ a smooth function. It is the purpose of this paper to explain to which extent this formal approximation is valid by proving estimates between the formal approximation $A_{\text {per }}\left[\psi\left(\delta X, \delta^{2} T\right), \phi_{0}\right](X)$ and exact solutions $A=A(X, T)$ of the real Ginzburg-Landau equation.

It turns out that we cannot expect validity uniformly for all $X \in \mathbb{R}$, but validity only uniformly for all $X \in I_{\delta}$ with $I_{\delta}$ an interval of length $O\left(\delta^{-r}\right)$ for all $r<2$.

Other amplitude equations for the evolution of the local wave number of stable and unstable planforms close to the Eckhaus-stable threshold in the Ginzburg-Landau equation have been considered in [Be88, vH95].

It is not obvious a priori that an approximation result for the phase diffusion equation (1.1) holds. There are a number of counterexamples of amplitude equations which are derived formally in a correct way, but do not describe the dynamics in the original system in a correct way. See for instance [Sch95].

The difficulty in justifying the phase-diffusion equation for the Ginzburg-Landau equation is the time scale $O\left(1 / \delta^{2}\right)$. Since the solutions in consideration are of order $O(1)$ a simple application of Gronwall's inequality would only give a time scale $O(1)$. In order to gain estimates on the long time scale $O\left(1 / \delta^{2}\right)$ the proof is built similarly to the derivation of the phase diffusion equation. In particular, in polar coordinates the equations for the error turn out to be quasilinear.

Our approximation result allows us to find the dynamics of the phase diffusion equation in the real Ginzburg-Landau equation. Moreover, the Ginzburg-Landau equation approximates more complicated pattern forming systems like the Taylor-Couette problem, close to the first instability, and so we can find the dynamics of the phase diffusion equation in these more complicated systems.

The dynamics of nonlinear diffusion equations like the phase diffusion equation is well studied. There are a number of self similar solutions [BKL94]. Moreover, in contrast to the original systems, the maximum principle holds.

Away from the threshold of the first instability, phase-diffusion equations for the evolution of the local wave number can be derived in order to describe spatial and temporal modulations of the fully developed spatially periodic pattern. Formally, they have been derived and discussed by several authors in a number of papers (cf. [CN84, CH93, EINP00, MP79, Ma90, NPL93, PN94, KP96]). There are also mathematically rigorous stability and diffusive mixing results which show that at least some of the dynamics predicted by the phase-diffusion equations can be found in the GinzburgLandau equation (cf. [CEE92, BK92, GM98]) or in the associated pattern forming 
systems (cf. [Sch98]). It is the purpose of further research to justify the phase-diffusion equations themselves away from the threshold of the first instability.

The plan of the paper is as follows. In Section 2 we derive the phase-diffusion equation by introducing polar coordinates $A=r e^{i \phi}$ and introducing $\psi=\partial_{X} \phi$. In Section 3 we prove estimates which hold uniformly in space for the variables $(r, \psi)$. In Section 4 we go back to the original $A$-variable which leads to the result that estimates which hold uniformly in space cannot be expected for the approximation of $A$. We also explain that the uniformity of the approximation can be improved by solving the equations for the higher order corrections. See Remark 4.2. We note that the alternative approach of [Me98, Me99], discussed briefly in Remark 3.5, shows that the derivation of the phase-diffusion equation can be made exact for a certain class of solutions if derivative terms of all orders are included (so that equation (1.1) becomes a pseudodifferential equation).

Notation. Throughout this paper many different constants are denoted with the same symbol $C$. We denote the space of $n$-times weakly differentiable local uniformly Sobolev functions with $H_{l, u}^{n}$. This Banach space is equipped with the norm

$$
\|u\|_{H_{l, u}^{n}}=\sup _{x \in \mathbb{R}}\|u(\cdot)\|_{H^{n}(x, x+1)} .
$$

For details we refer to [Sch99].

\section{Derivation of the phase-diffusion equation for the real Ginz- burg-Landau equation}

As already said, the purpose of this paper is to justify a phase-diffusion equation describing the evolution of the wave number $q$ of the spatially locally preferred planform for the real Ginzburg-Landau equation

$$
\partial_{T} A=\partial_{X}^{2} A+A-A|A|^{2}
$$

with $X \in \mathbb{R}, T \geq 0$, and $A(X, T) \in \mathbb{C}$. This equation possesses a family of stationary solutions

$$
A=A_{\text {per }}\left[q, \phi_{0}\right](X)=\sqrt{1-q^{2}} e^{i q X+i \phi_{0}}
$$

with $\phi_{0}, q \in \mathbb{R}$ as spatially locally preferred planform. To derive the phase diffusion equation we introduce polar coordinates

$$
A(X, T)=r(X, T) e^{i \phi(X, T)}
$$

in the real Ginzburg-Landau equation and obtain

$$
\left\{\begin{array}{l}
\partial_{T} r=\partial_{X}^{2} r+r-\left(\partial_{X} \phi\right)^{2} r-r^{3} \\
\partial_{T} \phi=\partial_{X}^{2} \phi+\frac{2\left(\partial_{X} r\right)\left(\partial_{X} \phi\right)}{r}
\end{array}\right.
$$

Besides the interpretation in the introduction, another interpretation will be that the phase-diffusion equation is derived to describe the dynamics close to a stationary pattern $A_{\text {per }}\left[q, \phi_{0}\right]$. We choose $(r, \phi)=(1,0)$ as the starting stationary pattern. For 
the general case see Remark 2.1 below. We introduce the deviations $(s, \phi)$ by setting $r=1+s$ to obtain

$$
\begin{aligned}
\partial_{T} s & =\partial_{X}^{2} s-2 s-\left(\partial_{X} \phi\right)^{2}-\left(\partial_{X} \phi\right)^{2} s-3 s^{2}-s^{3}, \\
\partial_{T} \phi & =\partial_{X}^{2} \phi+\frac{2\left(\partial_{X} s\right)\left(\partial_{X} \phi\right)}{1+s} .
\end{aligned}
$$

We can replace the equation for the phase $\phi$ by an equation for the local wave number $\psi=\partial_{X} \phi$ to obtain

$$
\left\{\begin{array}{l}
\partial_{T} s=\partial_{X}^{2} s-2 s-\psi^{2}-\psi^{2} s-3 s^{2}-s^{3} \\
\partial_{T} \psi=\partial_{X}^{2} \psi+2 \partial_{X}\left(\frac{\left(\partial_{X} s\right) \psi}{1+s}\right) .
\end{array}\right.
$$

The linearized system

$$
\begin{aligned}
\partial_{T} s & =\partial_{X}^{2} s-2 s, \\
\partial_{T} \psi & =\partial_{X}^{2} \psi,
\end{aligned}
$$

possesses solutions $(s, \psi)=\left(s_{k}, \psi_{k}\right) e^{i k x+\mu_{j}(k) t}$ with $\mu_{1}(k)=-k^{2}$, and $\mu_{2}(k)=-k^{2}-2$. Hence, the component $s$ which corresponds to $\mu_{2}$ is exponentially damped and so we expect $s$ to be slaved by $\psi$ which will behave diffusively. To derive the phase-diffusion equation we make a long wave ansatz

$$
\psi(X, T)=\check{\psi}\left(\delta X, \delta^{2} T\right) \quad \text { and } \quad s(X, T)=\check{s}\left(\delta X, \delta^{2} T\right)
$$

with $0 \leq \delta \ll 1$ a small perturbation parameter. This yields

$$
\left\{\begin{aligned}
\delta^{2} \partial_{\tau} \check{s} & =\delta^{2} \partial_{\xi}^{2} \check{s}-2 \check{s}-\check{\psi}^{2}-\check{\psi}^{2} \check{s}-3 \check{s}^{2}-\check{s}^{3} \\
\partial_{\tau} \check{\psi} & =\partial_{\xi}^{2} \check{\psi}+2 \partial_{\xi}\left(\frac{\check{\psi} \partial_{\xi} \check{s}}{1+\check{s}}\right)
\end{aligned}\right.
$$

with $\tau=\delta^{2} T$ and $\xi=\delta X$. Neglecting terms of order $O\left(\delta^{2}\right)$ for small $\check{\psi}$ by the implicit function theorem, the first equation

$$
0=-2 \check{s}-\check{\psi}^{2}-\check{\psi}^{2} \check{s}-3 \check{s}^{2}-\check{s}^{3}
$$

possesses a unique solution $\check{s}=s^{*}(\check{\psi})$ with $s^{*}(\breve{\psi})=-\check{\psi}^{2} / 2+O\left(\check{\psi}^{3}\right)$. (Due to the formula for the equilibria, we have in fact that $1+s^{*}=\sqrt{1-\psi^{2}}$.) Inserting this into the equation for $\tilde{\psi}$ leads finally to the phase diffusion equation

$$
\partial_{\tau} \check{\psi}=\partial_{\xi}^{2} \check{\psi}+2 \partial_{\xi}\left(\frac{\check{\psi} \partial_{\xi}\left(s^{*}(\check{\psi})\right)}{1+s^{*}(\check{\psi})}\right)=\partial_{\xi}^{2} h(\check{\psi})
$$

where $h: \mathbb{R} \rightarrow \mathbb{R}$ is smooth. Indeed, $h$ is the solution to the equation

$$
h^{\prime}(q)=1+\frac{2 q}{1+s^{*}(q)} s^{* \prime}(q)=\frac{1-3 q^{2}}{1-q^{2}} .
$$

Remark 2.1. Suppose that instead we start with a general choice of basic equilibrium $r=r_{q}, \phi=q X+\phi_{0}, q \in(-1,1)$. Then the corresponding phase diffusion equation is given by

$$
\partial_{\tau} \check{\psi}=\partial_{\xi}^{2} \tilde{h}(\check{\psi}, q)
$$


where $\tilde{h}(\check{\psi}, q)=h(\check{\psi}+q)$.

To verify this, note that the deviations $\check{\psi}_{q}$ for (2.8) are related to the deviations $\check{\psi}_{0}$ for $(2.7)$ by $\check{\psi}_{q}+q=\check{\psi}_{0}$. Hence $\partial_{\tau} \check{\psi}_{q}=\partial_{\tau} \check{\psi}_{0}=h\left(\check{\psi}_{0}\right)=h(\check{\psi}+q)=\tilde{h}(\check{\psi}, q)$ as required.

Remark 2.2. For each $q$, let $k \mapsto \mu_{1,2}(k, q)$ denote the smooth curves of eigenvalues corresponding to the Fourier wave numbers $k$ for the linearization of (2.3) around $(r, \phi)=\left(\sqrt{1-q^{2}}, q X\right)$. In particular, let $\mu_{1}(k, q)$ denote the critical curve for which $\mu_{1}(0, q)=0$. Then we claim that the phase-diffusion equation (2.7) must give at lowest order a linear diffusion equation with diffusion coefficient

$$
h^{\prime}(q)=-\partial_{k}^{2} \mu_{1}(0, q) / 2 .
$$

This is easily verified by direct calculation, since we have already seen that $h^{\prime}(q)=$ $\left(1-3 q^{2}\right) /\left(1-q^{2}\right)$ while $\mu_{1}(k, q)=-k^{2}-\left(1-q^{2}\right)+\sqrt{\left(1-q^{2}\right)^{2}+4 q^{2} k^{2}}$. A more conceptual proof is as follows.

First note that the linearization $L(q)$ of the right-hand-side $\partial_{X}^{2} \tilde{h}(\psi, q)$ of $(2.8)$ at $(0, q)$ must also have the eigenvalues $\mu_{1}(k, q)$ - after taking into account the fact that higher order derivatives have been neglected in the derivation of (2.8). But

$$
\partial_{X}^{2} \tilde{h}(\psi, q)=\partial_{X}^{2} h(q+\psi)=h^{\prime}(q) \partial_{X}^{2} \psi+O\left(|\psi|^{2}\right)
$$

and so $L(q)=h^{\prime}(q) \partial_{X}^{2}$. Equating $L(q) e^{i k X}=\mu_{1}(k, q) e^{i k x}$ modulo higher order derivatives yields $h^{\prime}(q)=-\partial_{k}^{2} \mu_{1}(0, q) / 2$ as required.

Note that $\partial_{k}^{2} \mu_{1}(0, q)>0$ if and only if $q^{2}>1 / 3$ which is the famous Eckhaus instability criterion [Eck65].

Remark 2.3. If the solutions of the phase-diffusion equation (2.7) start in between the Eckhaus stable range, i.e. having values $|\breve{\psi}|^{2}<1 / 3$, they stay in this Eckhaus stable range for all times due to the maximum principle. For sufficiently smooth initial conditions satisfying $\sup _{X \in \mathbb{R}}|\check{\psi}(X)|^{2}<1 / 3$ there is the local and global existence of solutions (cf. [Kn68, LSU68, Va92]).

Remark 2.4. It is common in the literature to consider generalized versions of the real Ginzburg-Landau equation with more complicated nonlinearities. For example, terms such as $i \partial_{X} A|A|^{2}$ and $|A|^{4} A$ are often included. More generally, terms of the form $A^{b_{1}} \bar{A}^{b_{2}}\left(i \partial_{X} A\right)^{b_{3}}\left(i \partial_{X} \bar{A}\right)^{b_{4}}$ are permitted provided $b_{1}-b_{2}+b_{3}-b_{4}=1$. In this case, writing $A=r e^{i \phi}, r=1+s, \psi=\partial_{X} \phi$, leads to a system of the following form in place of (2.4):

$$
\left\{\begin{array}{l}
\partial_{T} s=\partial_{X}^{2} s+f\left(s,\left(\partial_{X} s\right)^{2}, \psi\right), \\
\partial_{T} \psi=\partial_{X}^{2} \psi+\partial_{X}\left(\left(\partial_{X} s\right) g\left(s,\left(\partial_{X} s\right)^{2}, \psi\right)\right)
\end{array}\right.
$$

where $f$ and $g$ are smooth functions of $s, \psi,\left(\partial_{X} s\right)^{2}$.

This structure can be verified as follows. The symmetries $(A \mapsto \bar{A}, X \mapsto-X)$ and $A \mapsto e^{i c} A$ of the real Ginzburg-Landau equation lead to the symmetries $(\phi \mapsto$ $-\phi, X \mapsto-X)$ and $\phi \mapsto \phi+c$ for the $(s, \phi)$ equations. Hence, we obtain $\partial_{T} s=$ 
$\partial_{X}^{2} s+f\left(s,\left(\partial_{X} s\right)^{2}, \partial_{X} \phi\right), \partial_{T} \phi=\partial_{X}^{2} \phi+\left(\partial_{X} s\right) g\left(s,\left(\partial_{X} s\right)^{2}, \partial_{X} \phi\right)$. Writing $\psi=\partial_{X} \phi$ yields (2.9).

Now we can write $\tau=\delta^{2} T$ and $\xi=\delta X$ and neglecting small terms we reduce as before to the phase-diffusion equation

$$
\partial_{\tau} \check{\psi}=\partial_{\xi}^{2} \check{\psi}+\partial_{\xi}\left(\left(\partial_{\xi} s^{*}(\check{\psi})\right) g\left(s^{*}(\check{\psi}), 0, \check{\psi}\right)\right)=\partial_{\xi}^{2} h(\check{\psi}),
$$

where $h$ satisfies $h^{\prime}(q)=1+s^{* \prime}(q) g\left(s^{*}(q), 0, q\right)$. Again, $h^{\prime}(q)=-\partial_{k}^{2} \mu_{1}(0, q) / 2$ where $k \mapsto \mu_{1}(k, q)$ is the smooth critical curve of eigenvalues corresponding to Fourier wave numbers $k$. The estimates of this paper can be proved in a similar manner for this more general system, too.

\section{The approximation theorem for the $(\check{s}, \check{\psi})$-system}

In this section we prove that solutions of the $(\check{s}, \check{\psi})$-system $(2.5)$ can be approximated via solutions of the phase diffusion equation (2.7).

Theorem 3.1. Let $2 \leq m \leq n-2$. Then there exists a $C_{\psi}^{*}>0$ such that the following is true. Let $\psi^{*}$ be a solution of the phase diffusion equation (2.7) with

$$
\sup _{\tau^{\prime} \in\left[0, \tau_{0}\right]}\left\|\psi^{*}(\tau)\right\|_{H_{l, u}^{n}} \leq C_{\psi}^{*}
$$

and let $s^{*}$ be defined by the solution $s^{*}=s^{*}\left(\psi^{*}\right)$ of (2.6). Then there exist $\delta_{0}>0$ and $C_{2}>0$ such that for all $\delta \in\left(0, \delta_{0}\right)$ we have solutions $(\check{s}, \check{\psi})$ of the $(\check{s}, \check{\psi})$-system $(2.5)$ such that

$$
\sup _{\tau \in\left[0, \tau_{0}\right]}\left\|(\check{s}, \check{\psi})(\tau)-\left(s^{*}\left(\psi^{*}(\tau)\right), \psi^{*}(\tau)\right)\right\|_{H_{l, u}^{m} \times H_{l, u}^{m}} \leq C_{2} \delta^{2} .
$$

Proof . Let $\psi^{*}$ be a solution of the phase-diffusion equation (2.7) and let $s^{*}=s^{*}\left(\psi^{*}\right)$ be defined by (2.6) as above. We write a solution as approximation plus some error, i.e.

$$
\check{\psi}=\psi^{*}+\delta^{2} R_{\psi} \quad, \quad \check{s}=s^{*}+\delta^{2} R_{s} .
$$

We obtain

$$
\begin{aligned}
\partial_{\tau} R_{\psi} & =\partial_{\xi}^{2} R_{\psi}+L_{\psi}\left(R_{\psi}, R_{s}\right)+\delta^{2} N_{\psi}\left(R_{\psi}, R_{s}\right) \\
\partial_{\tau} R_{s} & =\partial_{\xi}^{2} R_{s}-2 \delta^{-2} R_{s}+\delta^{-2} L_{s}\left(R_{\psi}, R_{s}\right)+N_{s}\left(R_{\psi}, R_{s}\right)+\delta^{-2} \operatorname{Res}_{s}
\end{aligned}
$$

where

$$
\begin{aligned}
L_{\psi}\left(R_{\psi}, R_{s}\right) & =-2 \partial_{\xi}\left(\frac{\left(\partial_{\xi} s^{*}\right) R_{\psi}}{1+s^{*}}+\frac{\left(\partial_{\xi} R_{s}\right) \psi^{*}}{1+s^{*}}-\frac{\left(\partial_{\xi} s^{*}\right) \psi^{*}}{\left(1+s^{*}\right)^{2}} R_{s}\right) \\
\delta^{2} N_{\psi}\left(R_{\psi}, R_{s}\right) & =-2 \partial_{\xi}\left(\frac{\left(\partial_{\xi}\left(s^{*}+\delta^{2} R_{s}\right)\right)\left(\psi^{*}+\delta^{2} R_{\psi}\right)}{1+\left(s^{*}+\delta^{2} R_{s}\right)}\right)-L_{\psi}\left(R_{\psi}, R_{s}\right) \\
L_{s}\left(R_{\psi}, R_{s}\right) & =-2 \psi^{*} R_{\psi}-2 \psi^{*} s^{*} R_{\psi}-\left(\psi^{*}\right)^{2} R_{s}
\end{aligned}
$$




$$
\begin{aligned}
& -6 s^{*} R_{s}-3\left(s^{*}\right)^{2} R_{s} \\
N_{s}\left(R_{\psi}, R_{s}\right)= & -R_{\psi}^{2}-R_{\psi}^{2} s^{*}-2 R_{\psi} R_{s} \psi^{*}-\delta^{2} R_{\psi}^{2} R_{s} \\
& -3 R_{s}^{2}-3 s^{*} R_{s}^{2}-\delta^{2} R_{s}^{3} \\
\operatorname{Res}_{s}= & -\partial_{\tau} s^{*}+\partial_{\xi}^{2} s^{*}
\end{aligned}
$$

To be more precise, let

$$
\psi^{*} \in C\left(\left[0, \tau_{0}\right], H_{l, u}^{n}\right)
$$

be a solution of the phase-diffusion equation (2.7) for a $\tau_{0}>0$. Since $\partial_{\tau} \psi^{*}$ can be estimated by the right hand side of the phase-diffusion equation we have

$$
\partial_{\tau} \psi^{*} \in C\left(\left[0, \tau_{0}\right], H_{l, u}^{n-2}\right) .
$$

From the definition of $s^{*}$, we have

$$
s^{*} \in C\left(\left[0, \tau_{0}\right], H_{l, u}^{n}\right)
$$

and $\partial_{\tau} s^{*}, \partial_{\xi}^{2} s^{*} \in C\left(\left[0, \tau_{0}\right], H_{l, u}^{n-2}\right)$. Hence, for the residual term $\operatorname{Res}_{s}$ there exists a constant $C_{\text {Res }}$ independent of $\delta \in(0,1]$ such that

$$
\sup _{\tau \in\left[0, \tau_{0}\right]}\left(\left\|\operatorname{Res}_{s}(\tau)\right\|_{H_{l, u}^{n-2}}\right) \leq C_{\text {Res }} .
$$

A natural choice to solve the equations for the error $\left(R_{\psi}, R_{s}\right)$ would be the space $H_{l, u}^{m} \times H_{l, u}^{m+1}$. This choice would allow us to solve the equations as a semilinear system. However, it turns out that we have to build our proof similar to the derivation of the phase-diffusion equation (2.7) in order to gain enough powers of $\delta$. Due to the formula for $s^{*}=s^{*}\left(\psi^{*}\right)$ we will consider $\left(R_{\psi}, R_{s}\right) \in H_{l, u}^{m} \times H_{l, u}^{m}$ which leads to a quasilinear system. The semilinear approach fails due to the second estimate in Lemma 3.4, for the damped part, which is sharp with respect to powers of $\delta$.

Therefore, let us fix $m$ for the rest of this section satisfying $2 \leq m \leq n-2$ and assume $\tau \in\left[0, \tau_{0}\right]$.

Lemma 3.2. There exists a $C_{1}>0$ such that for all $R_{\psi}$ and $R_{s}$ and all $\delta \in(0,1]$ :

$$
\begin{aligned}
\left\|L_{\psi}\left(R_{\psi}, R_{s}\right)\right\|_{H_{l, u}^{m-2}} & \leq C_{1}\left(\left\|R_{\psi}\right\|_{H_{l, u}^{m}}+\left\|R_{s}\right\|_{H_{l, u}^{m}}\right), \\
\left\|L_{s}\left(R_{\psi}, R_{s}\right)\right\|_{H_{l, u}^{m}} & \leq C_{1}\left(\left\|R_{\psi}\right\|_{H_{l, u}^{m}}+\left\|R_{s}\right\|_{H_{l, u}^{m}}\right) .
\end{aligned}
$$

The constant $C_{1}>0$ can be chosen to satisfy $C_{1} \rightarrow 0$ if $\sup _{\tau \in\left[0, \tau_{0}\right]}\left\|\psi^{*}(\tau)\right\|_{H_{l, u}^{n}} \rightarrow 0$.

For all $M>0$ there exist $\delta_{1}, C>0$ such that for all $\delta \in\left(0, \delta_{1}\right)$ and $R_{\psi}$ and $R_{s}$ with $\left\|R_{\psi}\right\|_{H_{l, u}^{m}}+\left\|R_{s}\right\|_{H_{l, u}^{m}} \leq M$ we have

$$
\begin{array}{r}
\left\|\delta^{2} N_{\psi}\left(R_{\psi}, R_{s}\right)\right\|_{H_{l, u}^{m-2}} \leq C \delta^{2}\left(\left\|R_{\psi}\right\|_{H_{l, u}^{m}}^{2}+\left\|R_{s}\right\|_{H_{l, u}^{m}}^{2}\right), \\
\left\|N_{s}\left(R_{\psi}, R_{s}\right)\right\|_{H_{l, u}^{m}} \leq C\left(\left\|R_{\psi}\right\|_{H_{l, u}^{m}}^{2}+\left\|R_{s}\right\|_{H_{l, u}^{m}}^{2}\right) .
\end{array}
$$

Proof. Follows by elementary estimates as $\|u v\|_{H_{l, u}^{m}} \leq\|u\|_{H_{i, u}^{m}}\|v\|_{H_{l, u}^{m}}$ for $m \geq 1$. 
Lemma 3.3. The solution $R(\tau)=e^{\left(\partial_{\xi}^{2}-a\right) \tau} R_{0}$ of

$$
\partial_{\tau} R=\partial_{\xi}^{2} R-a R \quad,\left.\quad R\right|_{\tau=0}=R_{0}
$$

with $a \in \mathbb{R}$ satisfies for $r \geq 0$,

$$
\|R(\tau)\|_{H_{l, u}^{m+r}} \leq C e^{-a \tau}(1+\tau)^{-\frac{r}{2}}\left\|R_{0}\right\|_{H_{l, u}^{m}} .
$$

Proof . $\partial_{\xi}^{2}$ is a sectorial operator in $H_{l, u}^{m}$ generating the analytic semigroup $e^{\partial_{\xi}^{2} \tau}$. The estimates are standard in the theory of analytic semigroups (cf. [He81]) using multiplier theory in $H_{l, u}^{m}$-spaces as in [Sch99].

Since the nonlinear terms contain as many derivatives as the linear ones we need an optimal regularity result. We choose functions which are Hölder-continuous in time with values in $H_{l, u}^{m}$. We use the notation $\mu_{1}=\partial_{\xi}^{2}, \mu_{2}=\partial_{\xi}^{2}-2 \delta^{-1}, c_{1}=R_{\psi}$, and $c_{2}=R_{s}$ and consider for $j=1,2$

$$
\partial_{\tau} c_{j}=\mu_{j} c_{j}+g_{j}
$$

with

$$
g_{j} \in C^{\theta}\left(\left[0, \tau_{0}\right], H_{l, u}^{m}\right)
$$

and define

$$
C_{m, j}(\tau)=\left\|\left(c_{j}\left(\tau^{\prime}\right)\right)_{\tau^{\prime} \in[0, \tau]}\right\|_{C^{\theta}\left([0, \tau], H_{l, u}^{m}\right)}
$$

and similarly $C_{m, g_{j}}(\tau)$ for a $\theta \in(0,1)$.

Lemma 3.4. For $r \in[0,2]$ consider (3.3), where we additionally assume $g_{j}(0)=0$ for $2 \theta+r \geq 2$. Then the solutions $c_{j}$ of (3.3) satisfy

$$
C_{m+r, 1}(\tau) \leq C C_{m, g_{1}}(\tau) \quad \text { and } \quad C_{m+r, 2}(\tau) \leq C \delta^{2-r} C_{m, g_{2}}(\tau) .
$$

Proof. The proof follows by direct calculation based on a classical optimal regularity result (cf. [Am95]) using the estimates on the linear semigroup from above. In detail we have: For notational simplicity we first assume $g_{j}(0)=0$ and estimate with Lemma 3.3

$$
\begin{aligned}
& \left\|\int_{0}^{\tau} e^{\mu_{1}(k)\left(\tau-\tau^{\prime}\right)} g\left(\tau^{\prime}\right) d \tau^{\prime}\right\|_{H_{l, u}^{m+r}} \\
\leq & \left\|\int_{0}^{\tau} e^{\mu_{1}(k)\left(\tau-\tau^{\prime}\right)}\left(g\left(\tau^{\prime}\right)-g(\tau)\right) d \tau^{\prime}\right\|_{H_{l, u}^{m+r}}+\left\|\int_{0}^{\tau} e^{\mu_{1}(k)\left(\tau-\tau^{\prime}\right)} d \tau^{\prime} g(\tau)\right\|_{H_{l, u}^{m+r}} \\
\leq & C \int_{0}^{\tau} \tau^{\prime-r / 2} \tau^{\prime \theta} d \tau^{\prime} C_{m, g_{1}}(\tau)+\left\|\frac{1-e^{\mu_{1}(k) \tau}}{\mu_{1}(k)} g_{1}(\tau)\right\|_{H_{l, u}^{m+r}} \\
= & s_{1}+s_{2} .
\end{aligned}
$$

The first term $s_{1}$ is estimated by

$$
s_{1} \leq C \tau^{\theta} C_{m, g_{1}}(\tau) .
$$


In order to estimate $s_{2}$ we proceed as follows

$$
s_{2} \leq\left\|\frac{1-e^{\mu_{1}(k) \tau}}{\mu_{1}(k)}\right\|_{H_{l, u}^{m} \rightarrow H_{l, u}^{m+r}}\left\|g_{1}(\tau)\right\|_{H_{l, u}^{m}} .
$$

Since $g_{1}(0)=0$ we have

$$
\left\|g_{1}(\tau)\right\|_{H_{l, u}^{m}} \leq \tau^{\theta} C_{m, g_{1}}(\tau) .
$$

From the multiplier lemma in $H_{l, u}^{m}$-spaces (cf. [Sch99]) we have

$$
\left\|\frac{1-e^{\mu_{1}(k) \tau}}{\mu_{1}(k)}\right\|_{H_{l, u}^{m} \rightarrow H_{l, u}^{m+r}} \leq\left\|k \mapsto \frac{1-e^{\mu_{1}(k) \tau}}{\mu_{1}(k)}\left(1+|k|^{r}\right)\right\|_{C_{b}^{2}}
$$

which is estimated by

$$
\begin{aligned}
& \sup _{k \in \mathbb{R}}\left|\frac{1-e^{\mu_{1}(k) \tau}}{\mu_{1}(k)}\left(1+|k|^{r}\right)\right| \\
\leq & C\left(1+\sup _{|k| \geq 1} \mid \frac{|k|^{r}}{|k|^{2}}\right) \leq C
\end{aligned}
$$

and similarly for the derivatives. In a similar way we obtain

$$
\begin{aligned}
& \left\|\int_{0}^{\tau} e^{\mu_{2}(k)\left(\tau-\tau^{\prime}\right)} g\left(\tau^{\prime}\right) d \tau^{\prime}\right\|_{H_{l, u}^{m+r}} \\
\leq & C \int_{0}^{\tau} e^{-2 \delta^{-2} \tau^{\prime}} \tau^{\prime-r / 2} \tau^{\prime \theta} d \tau^{\prime} C_{m, g_{2}}(\tau)+\left\|\frac{1-e^{\mu_{2}(k) \tau}}{\mu_{2}(k)} g_{2}(\tau)\right\|_{H_{l, u}^{m+r}} \\
\leq & C \delta^{2-r} \tau^{\theta} C_{m, g_{1}}(\tau),
\end{aligned}
$$

where we introduced $\delta^{-2} \tau^{\prime}=\tilde{\tau}$ and estimated the integral by

$$
C \int_{0}^{\infty} e^{-\tilde{\tau}}\left(\delta^{2} \tilde{\tau}\right)^{-r / 2+\theta} \delta^{2} d \tilde{\tau}
$$

For the second term we use

$$
\sup _{|k| \geq 1}\left|\frac{k^{r}}{\delta^{-2}+k^{2}}\right| \leq \sup _{|k| \geq 1}\left|\frac{1}{\left(\delta^{-2}+k^{2}\right)^{1-r / 2}}\right| \sup _{|k| \geq 1}\left|\frac{k^{r}}{\left(\delta^{-2}+k^{2}\right)^{r / 2}}\right| \leq C \delta^{2-r} .
$$

These two estimates additionally show the Hölder-continuity with exponent $\theta$ for $\tau=0$. In a very similar fashion the Hölder-continuity for $\tau>0$ is obtained with the same estimates in terms of $\delta$ (cf. [Am95]). The estimates for $g_{j}(0) \neq 0$ and $2 \theta+r<2$ follow from classical theory for semilinear equations [He81].

Since the residual is arbitrarily smooth compared to $R_{\psi}$ and $R_{s}$, it can be handled with the semilinear estimate, i.e. $r=0$, and since $\left.R_{\psi}\right|_{\tau=0}=\left.R_{s}\right|_{\tau=0}=0$ implies $\left.L_{\psi}\right|_{\tau=0}=\left.L_{s}\right|_{\tau=0}=\left.N_{\psi}\right|_{\tau=0}=\left.N_{s}\right|_{\tau=0}=0$, Lemma 3.4 is applicable with $g_{1} \in\left\{L_{\psi}, N_{\psi}, \operatorname{Res}_{\psi}\right\}$ and $g_{2} \in\left\{L_{s}, N_{s}, \operatorname{Res}_{s},\right\}$. 
Hence it remains to sum up all estimates. Using (3.2), Lemma 3.2, and Lemma 3.4 gives finally the estimate

$$
\begin{aligned}
& C_{m, 1} \leq C_{4}\left(C_{m, 1}+C_{m, 2}\right)+C_{5} \delta^{2}\left(C_{m, 1}+C_{m, 2}\right)^{2}+C_{\text {Res }}, \\
& C_{m, 2} \leq C_{4}\left(C_{m, 1}+C_{m, 2}\right)+C_{5} \delta^{2}\left(C_{m, 1}+C_{m, 2}\right)^{2}+C_{\text {Res }}
\end{aligned}
$$

with $C_{4}$ a constant satisfying $C_{4} \rightarrow 0$ for $\sup _{\tau \in\left[0, \tau_{0}\right]}\left\|\psi^{*}(\tau)\right\|_{H_{l, u}^{n}} \rightarrow 0$, with $C_{5}$ another constant, both independent of $\delta$. From (3.4) and (3.5) for $\sup _{\tau \in\left[0, \tau_{0}\right]}\left\|\psi^{*}(\tau)\right\|_{H_{l, u}^{n}}>0$ and $\delta_{0}>0$ both sufficiently small we have

$$
C_{m, 1} \leq 2 C_{\mathrm{Res}} \quad \text { and } \quad C_{m, 2} \leq 2 C_{\mathrm{Res}}
$$

for all $\delta \in\left(0, \delta_{0}\right)$. Therefore, we are done.

Remark 3.5. An alternative approach [Me98, Me99] is to consider spaces of functions $s(X, T), \psi(X, T)$ that lie in a space $\mathcal{X}$ of Fourier transforms of Borel measures with bounded total variation norm. The space $\mathcal{X}$ is a Banach space with the total variation norm. We briefly describe the results of this approach, referring to [Me98, Me99] for details.

The starting point is the $(s, \psi)$ system (2.4) or more generally the system (2.9) obtained by including higher order terms in the real Ginzburg-Landau equation. It can be shown that locally there is a one-to-one correspondence between "essential solutions" for (2.9) and essential solutions for a pseudodifferential (in time and space) equation of the form $\partial_{T} \psi=\partial_{X}^{2} h+$ h.o.t where $h$ is the function that appears in (2.7) and h.o.t are higher order terms in the same sense as in the rest of this paper - of order $O\left(\delta^{3}\right)$ on writing $\tau=\delta^{2} T, \xi=\delta X$.

\section{The approximation theorem for the real Ginzburg-Landau equation}

It is the purpose of this final section to transfer the approximation result of Theorem 3.1 , i.e. that the $(\check{s}, \check{\psi})$-system $(2.5)$ can be approximated via solutions of the phase diffusion equation (2.7) back to the real Ginzburg-Landau equation (2.1). It turns out that we cannot expect validity uniformly for all $X \in \mathbb{R}$, but validity only uniformly for all $X \in I_{\delta}$ with $I_{\delta}$ arbitrary intervals of length $O\left(\delta^{-r}\right)$ for all $r<2$.

Our starting point is the relation

$$
A(X, T)=\left(1+\check{s}\left(\delta X, \delta^{2} T\right)\right) \exp \left(i \int_{0}^{X} \check{\psi}\left(\delta X^{\prime}, \delta^{2} T\right) d X^{\prime}\right)
$$

which defines the solution $A$ of the real Ginzburg-Landau equation (2.1) in terms of solutions $(\check{s}, \breve{\psi})$ of $(2.5)$. These solutions are approximated by

$$
A_{\text {app }}(X, T)=\left(1+s^{*}\left(\delta X, \delta^{2} T\right)\right) \exp \left(i \int_{0}^{X} \psi^{*}\left(\delta X^{\prime}, \delta^{2} T\right) d X^{\prime}\right) .
$$


Then we obtain

$$
\begin{aligned}
& \left|A(X, T)-A_{\text {app }}(X, T)\right| \\
\leq & \mid\left(1+\check{s}\left(\delta X, \delta^{2} T\right)\right) \exp \left(i \int_{0}^{X} \check{\psi}\left(\delta X^{\prime}, \delta^{2} T\right) d X^{\prime}\right) \\
& -\left(1+s^{*}\left(\delta X, \delta^{2} T\right)\right) \exp \left(i \int_{0}^{X} \psi^{*}\left(\delta X^{\prime}, \delta^{2} T\right) d X^{\prime}\right) \mid \\
\leq & \mid\left(1+\check{s}\left(\delta X, \delta^{2} T\right)\right) \exp \left(i \int_{0}^{X} \check{\psi}\left(\delta X^{\prime}, \delta^{2} T\right) d X^{\prime}\right) \\
& -\left(1+\check{s}\left(\delta X, \delta^{2} T\right)\right) \exp \left(i \int_{0}^{X} \psi^{*}\left(\delta X^{\prime}, \delta^{2} T\right) d X^{\prime}\right) \mid \\
& +\mid\left(1+\check{s}\left(\delta X, \delta^{2} T\right)\right) \exp \left(i \int_{0}^{X} \psi^{*}\left(\delta X^{\prime}, \delta^{2} T\right) d X^{\prime}\right) \\
\leq & \left|\int_{0} C \delta^{2} d X^{\prime}\right|+C \delta^{2} \leq C \delta^{2}(1+|X|) \\
\leq & C\left|\int_{0}^{X}\right| \check{\psi}\left(\delta X^{\prime}, \delta^{2} T\right)-\psi^{*}\left(\delta X^{\prime}, \delta^{2} T\right)\left|d X^{\prime}\right|+C \delta^{2} \\
+ & \\
\left.\left.+\mid s^{*}\left(\delta X, \delta^{2} T\right)\right)-\check{s}\left(\delta X, \delta^{2} T\right)\right) \mid & \exp \left(i \int_{0}^{X} \check{\psi}\left(\delta X^{\prime}, \delta^{2} T\right) d X^{\prime}\right)-\exp \left(i \int_{0}^{X} \psi^{*}\left(\delta X^{\prime}, \delta^{2} T\right) d X^{\prime}\right) \mid
\end{aligned}
$$

using the approximation result of Theorem 3.1. Thus, we have proved

Theorem 4.1. Under the assumptions of Theorem 3.1, there exists a solution $A=$ $A(X, T)$ of the real Ginzburg-Landau equation (2.1) such that the following is true. For all $L>0$ and $r \in[0,2)$ there exist $\delta_{0}>0$ and $C_{2}>0$ such that for all $\delta \in\left(0, \delta_{0}\right)$ we have

$$
\begin{aligned}
\sup _{T \in\left[0, \tau_{0} / \delta^{2}\right]} \sup _{|X| \leq L \delta^{-r}} \mid A(X, T)-\left(1+s^{*}\left(\psi^{*}\right)\left(\delta X, \delta^{2} T\right)\right) \\
\quad \times \exp \left(i \int_{0}^{X} \psi^{*}\left(\delta X^{\prime}, \delta^{2} T\right) d X^{\prime}\right) \mid \leq C_{2} \delta^{2-r}
\end{aligned}
$$


Hence the approximation result holds uniformly on intervals larger than the natural spatial scale $(r=1)$ of the phase diffusion equation. Due to the translation invariance of the original system this holds for all intervals of length $O\left(\delta^{-r}\right)$ using redefined approximations.

By taking $\check{\psi}=\delta^{2}$ and $\psi^{*}=0$ we have to compare $e^{i \delta^{2} X}$ with 1 which shows that estimates uniformly valid for all $X \in \mathbb{R}$ cannot be expected. A uniform estimate can only be expected for $\check{\psi}$ and $\psi^{*}$ spatially localized, for instance in spaces like $H^{m}(n)$ with $n \geq 2$, equipped with $\|u\|_{H^{m}(n)}=\left\|u \rho^{n}\right\|_{H^{m}}$ with $\rho(x)=\sqrt{1+x^{2}}$. We claim the validity of the approximation result for $(\check{\psi}, \check{s}) \in H^{m}(2) \times H^{m}(2)$, too.

Remark 4.2. By adding higher order terms in $\left(\psi^{*}, s^{*}\right)$, the approximation in Theorem 4.1 can be improved to hold uniformly on space intervals with length of order $O\left(\delta^{-p}\right)$ with $p \geq 0$ arbitrary. Indeed, it suffices to include all terms in $\left(\psi^{*}, s^{*}\right)$ of order $O\left(\delta^{\tilde{p}}\right)$ for some fixed $\tilde{p}>p$. In order to do so we make the ansatz

$$
\begin{aligned}
\check{\psi} & =\psi^{*}+\delta \psi_{1}^{*}+\delta^{2} \psi_{2}^{*}+\ldots+\delta^{\tilde{p}} \psi_{\tilde{p}}^{*}, \\
\check{s} & =s^{*}+\delta s_{1}^{*}+\delta^{2} s_{2}^{*}+\ldots+\delta^{\tilde{p}} \psi_{\tilde{p}}^{*} .
\end{aligned}
$$

The governing equations for the $\psi_{j}^{*}$ and $s_{j}^{*}$ are obtained by equating the coefficients of $\delta^{j+2}$ and $\delta^{j}$ to zero. They are linear with respect to $\psi_{j}^{*}$ and $s_{j}^{*}$ for fixed $j$. For instance, we obtain for $s_{1}^{*}$ the equation

$$
0=-2 s_{1}^{*}-2 \psi_{1}^{*} \psi^{*}-2 \psi_{1}^{*} \psi^{*} s^{*}-\left(\psi^{*}\right)^{2} s_{1}^{*}-6 s_{1}^{*} s^{*}-3\left(s^{*}\right)^{2} s_{1}^{*} .
$$

Hence the solutions of these equations exist as long as the solutions of the phase diffusion equation exist, again under the assumption that $\psi^{*}$ is sufficiently small.

\section{Acknowledgements}

Guido Schneider would like to thank A. Doelman, B. Sandstede and A. Scheel for stimulating discussions. The work of IAN MELBOURNE is partially supported by NSF Grant DMS-0071735. The work of GUIDO SCHNEIDER is partially supported by the Deutsche Forschungsgemeinschaft DFG under the grant Kr 690/18-1.

\section{References}

[Am95] H. Amann. Linear and quasilinear parabolic problems. Monographs in Mathematics. Birkhäuser 1995.

[Be88] A.J. BeRnoff. Slowly varying fully nonlinear wavetrains in the Ginzburg-Landau equation. Phys. D 30 (1988), no. 3, 363-381.

[BK92] J. Bricmont, A. Kupiainen. Renormalization group and the Ginzburg-Landau equation. Comm. Math. Phys. 150 (1992) 193-208.

[BKL94] J. Bricmont, A. Kupiainen, G. Lin. Renormalization group and asymptotics of solutions of nonlinear parabolic equations. Commun. Pure Appl. Math. 47 (1994) 893-922.

[CE90] P. Collet, J.-P. Eckmann. The time dependent amplitude equation for the SwiftHohenberg problem. Comm. Math. Phys. 132 (1990) 139-153.

[CEE92] P. Collet, J.-P. Eckmann, H. Epstein. Diffusive repair for the Ginsburg-Landau equation. Helv. Phys. Acta 65 (1992) 56-92. 
[CN84] M.C. Cross, A.C. Newell. Convection patterns in large aspect ratio systems. Physica D 10 (1984) 299-328.

[CH93] M.C. Cross, P.C. Hohenberg. Pattern formation outside of equilibrium. Rev. Mod. Phys. 65 (1993) 851-1090.

[Eck65] W. Eckhaus. Studies in nonlinear stability theory. Springer tracts in Nat. Phil. Vol. 6, 1965.

[EINP00] N.M. Ercolani, R. Indik, A.C. Newell, T. Passot. The geometry of the phase diffusion equation. J. Nonlinear Sci. 10 (2000) 223-274.

[GM98] T. Gallay, A. Mielke. Diffusive mixing of stable states in the Ginzburg-Landau equation. Comm. Math. Phys. 199 (1998) 71-97.

[He81] D. HenRY. Geometric Theory of Semilinear Parabolic Equations, LNM 840, Springer, BerlinNew York 1981.

[Kn68] B.F. KNERR. The porous medium equation in one dimension. Trans. Amer. Math. Soc. 234 (1977) 381-415.

[KP96] L. Kramer, W. Pesch. Electrohydrodynamic instabilities in nematic liqud crystals. In "Pattern Formation in Liquid Crystals" (Editors A.Buka und L.Kramer), Springer-Verlag, 1996, 221-255.

[LSU68] O.A. Ladyzhenskaja, V.A. Solonnikov, N.N. Ural'CeVA, Linear and quasi-linear equations of parabolic type. American Mathematical Society, Providence, R.I. 1968.

[MP79] P. Manneville, Y. Pomeau. Stability and fluctuations of a spatially periodic convection flow. J. Phys. Lett. 40 (1979) 609-612.

[Ma90] P. Manneville. Dissipative structures and weak turbulence. Perspectives in Physics. Boston, MA: Academic Press, Inc. xvii, 485 p. (1990).

[Me98] I. Melbourne. Derivation of the time-dependent Ginzburg-Landau equation on the line. J. Nonlinear Sci. 8 (1998) 1-15.

[Me99] I. Melbourne. Steady-state bifurcation with Euclidean symmetry. Trans. Am. Math. Soc. 351 (1999) 1575-1603.

[NPL93] A. Newell, T. Passot, J. Lega. Order parameter equations for patterns. Annu. Rev. Fluid Mech. 25 (1993) 399-453.

[PN94] T. Passot, A.C. Newell. Towards a universal theory of patterns. Physica D 74 (1994) 301-352.

[Sch94] G. SChNEIDER. Error estimates for the Ginzburg-Landau approximation. J. Appl. Math. Physics (ZAMP) 45 (1994) 433-457.

[Sch95] G. SCHNEIDER. Validity and Limitation of the Newell-Whitehead equation. Mathematische Nachrichten 176 (1995), 249-263.

[Sch98] G. SCHNEIDER. Nonlinear stability of Taylor-vortices in infinite cylinders. Arch. Rational Mech. Analysis 144 (1998) 121-200.

[Sch99] G. SCHNEIDER. Global existence results for pattern forming processes in infinite cylindrical domains. - Applications to 3D Navier-Stokes problems -. J. Mathématiques Pures Appliquées 78 (1999) 265-312.

[vH91] A. van HARTEN. On the validity of Ginzburg-Landau's equation. J. Nonlinear Science 1 (1991) 397-422.

[vH95] A. van Harten. Modulated modulation equations. Proceedings of the IUTAM/ISIMM Symposium on Structure and Dynamics of Nonlinear Waves in Fluids (Hannover, 1994), 117-130, Adv. Ser. Nonlinear Dynam., 7, World Sci. Publishing, River Edge, NJ, 1995.

[Va92] J.L. VAzQuez. An introduction to the mathematical theory of the porous medium equation. Shape optimization and free boundaries (Montreal, PQ, 1990), 347-389, NATO Adv. Sci. Inst. Ser. C Math. Phys. Sci., 380, Kluwer Acad. Publ., Dordrecht, 1992. 
IAN MELBOURNE,

Department of Mathematics and Statistics, University of Surrey,

Guildford, Surrey, GU2 7XH,

United Kingdom
GUIDO SCHNEIDER,

Mathematisches Institut I,

Universität Karlsruhe,

76128 Karlsruhe,

Germany. 